\title{
The extent of endoscopic sinus surgery in patients with severe chronic rhinosinusitis with nasal polyps (AirGOs Operative)*
}

\author{
Markus J. Lilja'1,2, Paula Virkkula', Sari Hammarén-Malmi' , Anu Laulajainen- \\ Hongisto', Lena Hafrén', Paula Kauppi², Johanna Sahlman³, Wytske J. \\ Fokkens ${ }^{4}$, Sietze Reitsma ${ }^{4}$, Sanna K. Toppila-Salmi²,5 \\ 'Department of Otorhinolaryngology and Head and Neck Surgery, University of Helsinki and Helsinki University Hospital, \\ Helsinki, Finland \\ 2 Department of Allergy, University of Helsinki and Helsinki University Hospital, Helsinki, Finland \\ ${ }^{3}$ Department of Otorhinolaryngology, University of Eastern Finland and Kuopio University Hospital, Kuopio, Finland \\ ${ }^{4}$ Department of Otorhinolaryngology, Amsterdam UMC, University of Amsterdam, the Netherlands \\ ${ }^{5}$ Haartman Institute, University of Helsinki, Helsinki, Finland
}

Rhinology Online, Vol 4: 154 - 160, 2021

http://doi.org/10.4193/RHINOL/21.029

*Received for publication:

June 23, 2021

Accepted: August 11, 2021

Published: August 23, 2021

\begin{abstract}
Background: Chronic rhinosinusitis with nasal polyps (CRSwNP) is a chronic inflammatory disease of the nose and paranasal sinuses characterized by intense inflammation, decreased health-related quality of life (HRQoL), and in severe cases high frequency of co-morbidities and recurrence despite treatment. Conservative treatment consists of nasal lavage, intranasal corticosteroids, and courses of oral corticosteroids, and antibiotics in exacerbations. Endoscopic sinus surgery (ESS) and/or biological therapy is considered if appropriate conservative treatment is not sufficient. The optimal extent of ESS in recalcitrant CRSwNP is not known. The aim of this randomized controlled trial is to evaluate and compare the efficacy and safety of limited ESS with partial ethmoidectomy with extended ESS with total ethmoidectomy in patients with severe CRSwNP.
\end{abstract}

Methods: AirGOs Operative is a randomized controlled trial. It is an investigator-driven multicenter trial led by Helsinki University Hospital. The two surgery arms are compared. The primary outcome is the change in the SNOT-22 score at the 12-month follow-up. Secondary outcomes include the change in the SNOT-22 score at 24-months follow-up, the changes in polyp score, Lund-Mackay (LM) CT score, health-related quality of life (HRQoL), loss of productivity, nasal patency (peak nasal inspiratory flow (PNIF) \pm acoustic rhinometry (ARM), olfaction test (Sniffin' Sticks, identification), lung function (spirometry and PEF) and findings in pathological analysis at 12/24-months follow-up.

Discussion: AirGOs Operative trial will lead to a better understanding of the optimal extent of ethmoidectomy in the treatment of recalcitrant severe CRSWNP.

Trial registration: ClinicalTrials.gov, NCT03704415. Registered on 8 of October 2018

Key words: chronic rhinosinusitis, computed tomography, nasal polyp, sinusitis, QoL

\section{Introduction}

Chronic rhinosinusitis (CRS) is an inflammatory disease of the nose and paranasal sinuses lasting for at least 12 weeks and with an incidence varying between $3-11 \%^{(1,2)}$. CRS is divided into two main phenotypes, CRS with nasal polyps (CRSwNP) and without (CRSsNP). The prevalence of CRSwNP is reported to be between $0,5-4,5 \%$ in the general population ${ }^{(3)}$. CRSwNP is characterized by intense inflammation, decreased health-related quality of life (HRQoL) and in severe cases recurrence despite treatment. It has a significant impact on airway health, and causes both direct and indirect costs ${ }^{(3,4)}$. The baseline treatment of CRSwNP contains nasal lavage and intranasal corticosteroids. In addition, courses of oral corticosteroids and antibiotics may be used in uncontrolled and/or exacerbated CRSwNP. Endoscopic sinus 
surgery (ESS) and/or biological therapy is considered if the appropriate conservative treatment fails.

ESS is an effective, safe, and a widely accepted surgical approach. ESS is individually tailored based on the clinical situation and surgeon's opinion, as well as published evidence and recommendations ${ }^{(3)}$. For CRSwNP patients the extent of ESS may vary from a simple polypectomy to extended ESS, such as removal of nasal middle turbinate and/or total ethmoidectomy and/or opening of all other nasal sinuses.

There is lacking knowledge of the optimal extent of ESS in recalcitrant CRSwNP. Mastersson et al. (2010) has reported that extensive ESS involving complete removal of nasal polyps along with a full ethmoidectomy had lower revision surgery rates compared to polyposis surgery limited to the anterior ethmoids ${ }^{(5)}$. Jankowski et al. (2006) have found that the endoscopic appearance of the mucosa was significantly better in radical ethmoidectomy (nasalization = opening of all sinuses, removal of mucosa on the orbital wall and ethmoid roof, resection of middle turbinate) compared with functional ethmoidectomy, adapted to the extent of pathology, five years after surgery. In addition, the overal nasal functional benefit was better, and the total recurrence rate was lower in the radical ethmoidectomy group ${ }^{(6)}$. In the study of Chen et al. (2016), the subjective olfaction and endoscopic appearance were better after extensive endoscopic sinus surgery (polypectomy, resection of inferior two-thirds of both middle turbinate and superior turbinate, total ethmoidectomy, antrostomies of maxillary, frontal, and sphenoid sinuses) compared with functional targeted (Messerklinger technique) endoscopic sinus surgery at one-year follow-up ${ }^{(7,8)}$. Zhang et al. (2020) have also reported, in a five-year prospective study, a lower long-term revision rate and a longer time to recurrence post-surgery in radical ESS group (full (F)ESS including Draf IIA frontal sinusotomy, and resection of the inferior two-third of middle turbinates) compared with full functional ESS (Messerklinger technique)

(9). However, most of these studies entail small groups, are not blinded and/or randomized, have short follow-up periods, could compare surgeons instead of surgeries, vary greatly in postoperative management, and thus carry a severe risk of bias.

Our nation-wide study has shown a large regional variation in the ESS rates in Finland, explained only in part by patients' age and differing availability of medical services ${ }^{(10)}$. There is some evidence that the recurrence rates of ESS may be lower after ESS with extended approaches compared with limited ones, but these studies are heterogeneous ${ }^{(5-7,9)}$. The weight of evidence for extended approaches lies in revision cases, not in primary surgeries ${ }^{(3)}$. Browne et al. (2006), in a multicenter cohort study, has concluded that nasal polypectomy with additional surgery seems to have no benefit over simple polypectomy in terms of health-related quality of life (HRQoL) improvement ${ }^{(11)}$. However, a prospective randomized comparison of limited partial ethmoidectomy and extended functional mucosa sparing ESS in medically recalcitrant CRSwNP is still lacking. A Japanese group showed in a database analysis of 50,734 patients, that the extent of ESS did not significantly affect the overall complication rate (12). However, extended surgery requires more operating time and is more expensive ${ }^{(12,13)}$. Potential disadvantages, financial implications, and available hospital resources should be considered when choosing the extent of ESS.

As there are unresolved questions concerning the ideal extent of ESS, the aim of this randomized controlled trial is to evaluate and compare the efficacy and safety of limited ESS with partial ethmoidectomy and extended ESS with total ethmoidectomy in patients with severe CRSwNP. The hypothesis is that extended sinus surgery is superior to limited sinus surgery as measured by validated $\mathrm{HRQ}$ oL questionnaires, endoscopic nasal polyp score, and CRS and asthma control. In addition, genome-wide molecular factors and microbiome are evaluated from nasal samples.

\section{Materials and methods}

\section{Primary objective}

The primary aim of this study is to evaluate the relative change of a validated Quality of Life score (Sinonasal Outcome Test 22; SNOT-22) at twelve months follow-up verses baseline in the two randomized operative treatment arms: (i) extended sinus surgery (ii) limited sinus surgery in patients with CRSwNP. Extended sinus surgery means total ethmoidectomy with opening of all nasal sinuses under general anesthesia, whereas limited sinus surgery means removal of polyps, uncinectomy, and partial ethmoidectomy primarily in local anesthesia with sedation, but in general anesthesia if necessary (e.g., patient preference, not a randomized choice between local and general anesthesia for partial ethmoidectomy). Partial ethmoidectomy was defined as the removal of lower anterior and posterior ethmoidal cells when frontal recess cells and cells adjacent to the skull base were intentionally left intact.

\section{Secondary objectives}

1. To evaluate the relative change (e.g. baseline vs. at 24 months postoperative) of SNOT-22 score in the two treatment arms

2. To evaluate the relative change of endoscopic nasal polyp score in the two treatment arms

3. To evaluate the relative change of the olfaction and nasal patency measurements in the two treatment arms

4. To evaluate the relative change of the asthma specific HRQoL (ACT, asthma control test) in the two treatment arms in those with asthma

5. To evaluate the relative change of lung function test results and exhaled $\mathrm{NO}$ in the two treatment arms

6. To evaluate exacerbation rates, need for rescue therapy, 


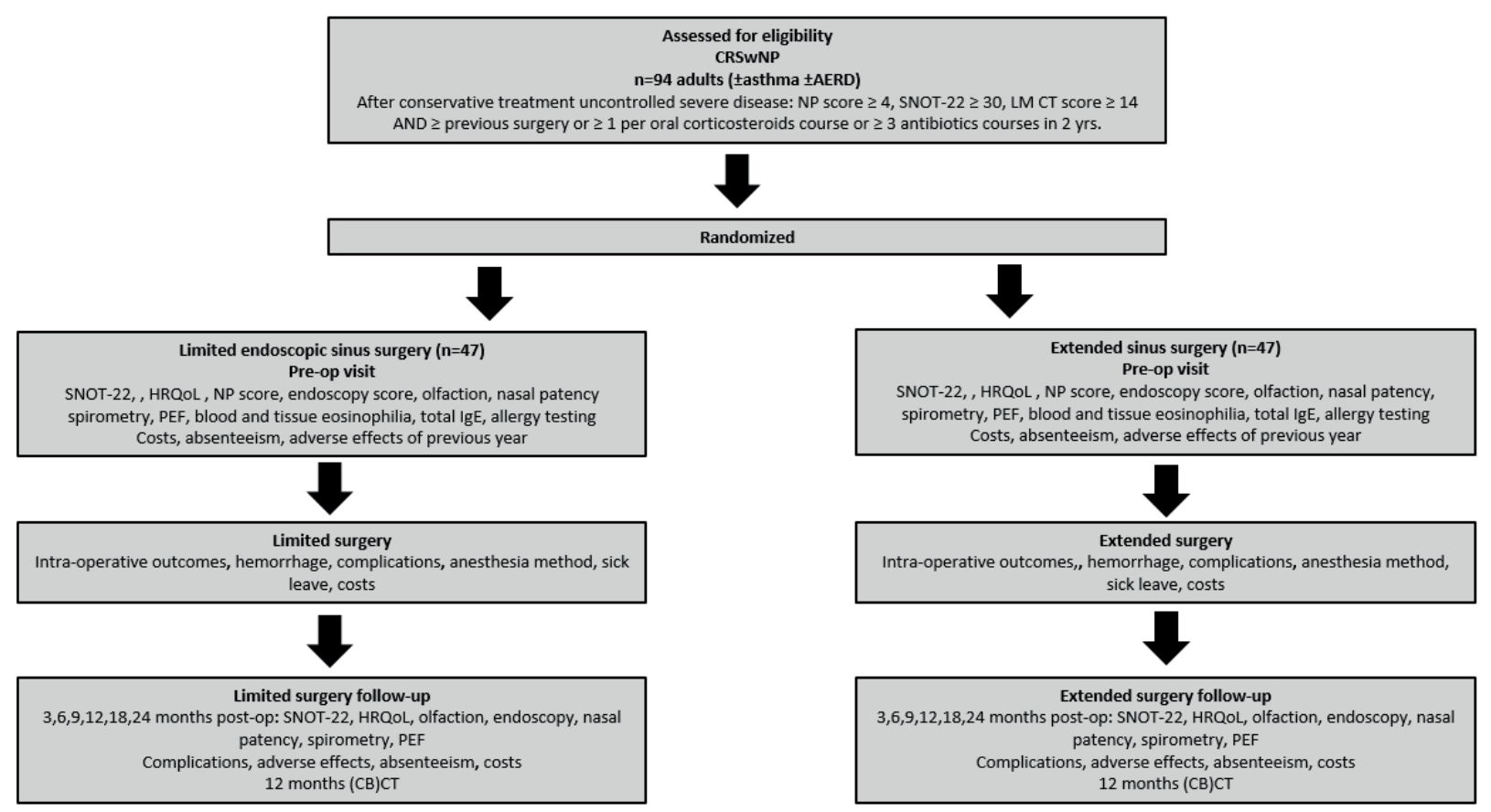

Figure 1. Flow diagram of AirGOs operative study. CB cone beam, CT computed tomography, HRQoL health-related quality of life, LM Lund-Mackay, NP nasal polyp, PEF peak expiratory flow, yrs years.

work absence, costs and safety in the two treatment arms

7. To evaluate intraoperative factors such as equipment needed, duration of surgery, the amount of bleeding, measures taken to control bleeding, and complications of in the two treatment arms.

8. To evaluate changes in molecular factors and microbiome in the two treatment arms

9. To identify biomarkers that predict postoperative control of CRSwNP (blood and tissue eosinophilia).

\section{AirGOs Operative trial design}

AirGOsOperative is a randomized controlled trial. It is an investigator-driven multicenter trial led by Helsinki University Hospital. Two countries involving 3 centers are participating in the AirGOsOperative trial. Patient enrollment started in 2018 and is expected to end in 2022. The study was approved by the local Research Ethics Boards (REBs) of the participating sites.

Figure 1 summarizes the design of the trial and each of the trials aspects is described in detail below.

\section{Eligibility criteria}

The inclusion criteria for patients evaluated for surgery are: age $\geq 18$ years, total endoscopic nasal polyp score ( $\geq 4$, bilateral), SNOT-22 $\geq 30$, and Lund-Mackay score of sinus computed tomography or sinus cone beam tomography scans (LM score 214). In addition, patients should have a history of at least one of the following: one previous surgery for CRSWNP; one or more oral corticosteroid course(s) during the past two years; three or more antibiotic courses during the past two year. In patients with contraindication/adverse effects for using oral steroids, the additional criteria of oral steroids are not required. All patients should meet the EPOS (European position paper on rhinosinusitis and nasal polyps 2012) criteria for chronic rhinosinusitis (14). Conservative treatment consists of corticosteroid drops and nasal lavage lasting at least 3 months prior to evaluation.

If the patient is excluded from participation, the reason for exclusion is recorded. The exclusion criterias are: age under 18 or above 65 years, complication of CRS (e.g. mucocele, invasive fungal rhinosinusitis), other diagnosis than CRSwNP (e.g. inverted papilloma, antrochoanal polyp), previous external sinus surgery or Draf III procedure or indication for external approaches or Draf III, bleeding diathesis, pregnancy or breastfeeding, cystic fibrosis, primary ciliary dyskinesia (PCD), sarcoidosis, granulomatosis with polyangitis (GPA), eosinophilic granulomatosis with polyangitis (EGPA), immunosuppression (diagnosed Specific Antibody Deficiency (SAD), common variable immunodeficiency (CVID), human immunodeficiency virus (HIV), or use of biologicals/immunosuppressive medication), immunotherapy, daily use of systemic corticosteroids, communication problems (e.g. neurological/psychiatric disease, language skills), unlikely to comply with the requirements of the trial, other severe disease, and inability to be operated. The patients are not allowed to use systemic corticosteroids during the previous month before the baseline visit. 
The study centers will screen all their CRSwNP patients and will document the screening failures to minimize bias in patient selection. Patients meeting inclusion criteria are given detailed oral and written information about the study. Participating patients will be randomized into two surgical arms. Of those declining participation, the age and gender distribution data are used into analyze whether the data is generalizable to the target population.

\section{Randomization}

Patients who meet the eligibility criteria are randomized to the two treatment arms with ratio of randomization of 1:1. A professional statistician from the University of Helsinki has developed a randomized sequence for the patients in both study arms. The randomization codes are in sealed envelopes, these envelopes are opened at the randomization visit. Blinding is not possible due to (i) putative difference in anesthesia methods of the treatment arms (ii) electronic health record data automatically released to the patient (https://www.kanta.fi/).

1. Extended sinus surgery -arm

In this arm, the patients undergo navigator-assisted ESS, including removal of polyps, and the opening of all nasal sinuses under general anesthesia with Draf IIA frontal sinusotomy. The sinus mucosa and middle turbinates are spared.

2. Limited endoscopic sinus surgery-arm Patients undergo removal of polyps, uncinectomy with or without middle meatal antrostomy and partial ethmoidectomy preferably in local anesthesia.

Clinicians and patients are not blinded to the treatment arm in this trial. Experienced rhinological surgeons perform surgery in both treatment groups. Surgeries will be performed using powered instrumentation (microdebrider).

\section{Follow-up}

Patients are followed every three months for 24 months after surgery. Detailed information on the content of the follow-up is presented in Figure 1.

\section{Definition of CRSWNP and co-morbidities}

The CRSwNP phenotype is defined according to EPOS 2020 criteria, by a history of nasal polyps and endoscopic signs of nasal polyps ${ }^{(3)}$. Clinical data of previous polyp surgery, time of diagnosis of nasal polyposis, allergies, asthma diagnosis, NSAID (non-steroidal anti-inflammatory drug) exacerbated respiratory disease (NERD), profession, work environment, smoking habits, medication and disease history are recorded by the investigator. The diagnosis of asthma is based on typical history, clinical features, and at least one of the following physiologic criteria: (i) a variation of $20 \%$ or greater in diurnal peak expiratory flow (PEF) recording (reference to diurnal mean); (ii) an increase of $15 \%$ or greater in PEF with $\beta$-agonist or (iii) an increase of $12 \%$ or greater in forced expiratory volume in 1 second (FEV1) with $\beta$-agonist; or (iv) a decrease of $15 \%$ or greater in PEF or FEV1 in exercise testing or moderate to severe bronchial hyperresponsiveness. NERD diagnosis is based on a positive history of wheeze/ cough or naso-ocular symptoms after intake of NSAID and/or positive ASA challenge. Allergy is verified with positive history and Skin Prick Test and/or serum allergen-specific lgE against common aeroallergens.

\section{Results}

Primary and secondary outcomes

The primary outcome is the change in SNOT-22 score at 12-months follow-up. Secondary outcomes include change in SNOT-22 score at 24-months follow-up, changes in polyp score, LM CT score, HRQoL, loss of productivity, nasal patency (peak nasal inspiratory flow (PNIF) \pm acoustic rhinometry (ARM), olfaction test (Sniffin' Sticks, identification), lung function (spirometry, exhaled NO, and PEF) and findings in pathological analysis at 12/24-months follow-up (Figure 1). Safety (complications, adverse effects), costs and loss of productivity will be compared between the study arms. Also the costs of CRSWNP and asthma treatment one year before and two years after surgery will be compared, taking into account study arms.

\section{Health related quality of life (HRQoL)}

Disease-specific quality of life is monitored with the SNOT-22, which is a widely used instrument designed to measure a wide range of consequences of CRS including physical, functional and emotional aspects. It has been shown to be reliable and useful tool to show intervention-related changes ${ }^{(15,16)}$. Two selfadministered questionnaires of general HRQoL are included: 1. EQ-5D-5L includes fives questions about mobility, self-care, usual activities, pain or discomfort and anxiety or depression. An EQ-5D-5L index is calculated and gives a patient's health status on a scale ranging from 0 (very bad health) to 1 (perfect health). Patients also rate their overall generic HRQoL using a Visual Analogue Scale (EuroQoL-5D VAS) from 0 (very bad health) to 100 (perfect health). 2. The 15D questionnaire is a standardized, validated $\mathrm{HRQOL}$ instrument that can be used both as a profile and a single-index score ${ }^{(17)}$. The questionnaire consists of 15 dimensions (15D): moving, seeing, hearing, breathing, sleeping, eating, speech, excretion, normal activities, mental functioning, discomfort and symptoms, depression, distress, vitality, and sexual activity. For each dimension, the respondent must choose one of the five levels that best describes her/his state of health at that moment (best level $=1$; poorest level $=5$ ). The value of $15 \mathrm{D}$ is based on applying the multi-attribute utility theory. The single index score (15D score), representing the overall 
HRQoL on a scale from 0 to 1 scale ( $1=$ full health, $0=$ being dead) and the dimension level values reflecting the goodness of levels relative to no problems along a dimension and to being dead $(0)$ are calculated from the questionnaire using a set of population-based preference or utility weights. Mean dimension level values are used to create 15D profiles for groups. The generic, clinically important minimal difference (MID) in the 15D scores is $\pm 0.015^{(18)}$.

\section{Olfactory function}

Olfaction is measured with Sniffing Sticks Identification Test;its twelve pens contain common smells and during the test, one pen is held under the nose for a few seconds at a time. A forced choice out of four options is inquired after explaining that this may well increase the possibility of correct answers by chance. The score of correct answers per 12 smells is counted.

\section{Nasal patency}

Nasal patency is measured by the peak nasal inspiratory flow (PNIF) method using a portable GMI PNIF meter (GM Instruments). A ventilation mask covers the nose firmly and the patient is asked to close the mouth and to inhale through the nose as quickly as possible. The highest inspirations speed (L/min) of three maximal inspirations is accepted for analysis.

Acoustic rhinometry gives an estimate of the cross-sectional diameter of the nasal cavity at each distance, minimal crosssectional areas are recorded. Acoustic rhinometry is performed after an acclimatization period of 15-20 minutes to allow adaptation to the room temperature and humidity. A trained nurse or doctor performs the measurements.

\section{Nasal endoscopy}

Modified Lund endoscopy score is used to evaluate polyps, oedema and discharge on a score from 0 to $2^{(19)}$. The polyps are also scored on a four point scale with modified Davos scale from 0 to 4 on each side (score 0 : no polyps, score 1 : small polyps in the middle meatus not reaching below the inferior border of the middle turbinate, score 2: polyps in the middle meatus reaching below the inferior border of the middle turbinate, score 3: polyps are below the lower border of the middle turbinate and the medial polyps are large or any polyp reaches the lower border of inferior turbinate, score 4: the polyps reach below the lower border of the inferior turbinate) ${ }^{(20)}$. A total count of the sides is reported.

\section{CRS disease control}

CRS control criteria have been defined in EPOS 2012 and 2020

${ }^{(3,14)}$. Disease control is defined by the common nasal symptoms and sleep disturbance or fatigue, endoscopy findings and need for systemic corticosteroids or long-term antibiotics during the previous one or three months ${ }^{(14,21)}$. Control of symptoms is defined as a disease state in which the patients do not report symptoms, or the symptoms are not bothersome.

\section{Asthma control}

Asthma control assessed with Asthma Control Test (ACT) and Mini Asthma Quality of Life Questionnaire (Mini AQLQ). ACT assesses the frequency of shortness of breath and general asthma symptoms, use of rescue medications, the effect of asthma on daily functioning, and overall self-assessment of asthma control. Symptoms and activities are assessed with 5-point scale: $1=$ all the time to $5=$ not at all; for asthma control rating: $1=$ not controlled at all to $5=$ completely controlled). The scores range from 5 (poor control of asthma) to 25 (complete control of asthma), with higher scores reflecting greater asthma control. An ACT score $>19$ indicates well-controlled asthma. The Mini AQLQ instrument has 15 questions in the same domains as the original AQLQ (symptoms, activities, emotions and environment). A change in score of greater than 0.5 is considered clinically important.

\section{Exacerbations}

Exacerbations of upper or lower airway symptoms which need increased medication, emergency or outpatient visits, hospitalization, as well as decreased productivity and sick leaves are recorded during the previous one year before ESS and during each follow-up contact during the 24-months follow-up. The patients report impaired activity and productivity with Work Productivity and Activity Questionnaire (WPAI:GH).

\section{Productivity, health resource use and costs}

The use of resource and the costs of health care utilization, medical costs, and productivity are obtained from hospital databases, intervention reports, medical files and national health records. This data is also collected with the Productivity COST Questionnaire (iPCQ) and Medical Consumption Questionnaire (iMCQ) ${ }^{(22,23)}$.

\section{Adverse effects and safety}

Adverse effects of CRS and asthma treatment during the past year before enrollment and at every visit and call during 24-months follow-up are inquired. Complications of surgery are recorded during the perioperative period in the hospital and during follow-up. Sudden adverse effects and sudden unexpected adverse effects during the trial are recorded as recommended by the Guideline for Good Clinical Practice (GCP) updated in 2017(EMA/CPMP/ICH/135/95).

\section{Tissue and mucus samples}

Nasal mucus, epithelial and polyp tissue samples are obtained before the operation and 12 months after surgery to investigate eosinophilia, and in part of the patients microbiome and trans- 
criptome profiles associated with ESS outcomes. The Finnish patients are asked to voluntarily donate a blood sample to the Finnish national Biobank for a national genomi-wide association study (GWAS) project (https://www.finngen.fi/en).

\section{Data collection, management and storage}

Clinical data is collected from electronic patient files and paper questionnaires are used to retrieve study data. Paper questionnaires, results of measurements, and signed informed consents are stored in locked cabinets. The collected data is stored for analysis in electronic Case Report Forms, a storage system provided for Helsinki University Hospital by Granitics Ltd.

\section{Sample size}

The mean postoperative difference in the SNOT-22 was assumed to be at least nine between the treatment arms. In a previous study, the response within each subject group was normally distributed with a standard deviation of 15 . If the true difference in the experimental and control means is nine, we will need to study 45 experimental subjects and 45 control subjects to be able to reject the null hypothesis that the population means of the experimental and control groups are equal with a probability (power) of 0,8 . The Type I error probability associated with this test of this null hypothesis is 0,05 . With the estimated drop-out-rate of $5 \%$, the minimum number of patients needed to recruit is 94 .

\section{Statistical methods}

The study's primary objective is improvement in the endpoints of change from baseline to 1 year postoperative in SNOT22 scores, endoscopic nasal polyp (NP) score. Power calculations for the primary endpoints and procedures for randomization were detailed previously. Change from baseline at 1 year postoperatively in FEV1 in the subset of patients (at Helsinki University Hospital) is an additional secondary endpoint. Each of the imputed complete datapoints is analyzed by fitting an analysis of covariance (ANCOVA) model with the corresponding baseline value, treatment group, prior surgery history, sex, age and region as covariates.

Monitoring, safety and reporting of adverse effects The study is monitored by the monitoring unit of the Hospital District of Helsinki and Uusimaa, and an independent monitoring committee of the Clinical Research Unit at the Academic Medical Centre. The monitoring is conducted according to the International Conference of Harmonization guidelines. Monitoring ensures that the rights and well-being of the participants are protected, that the recorded data is accurate and verifiable from the source documents, and that the study protocol, GCP and other regulations concerning clinical studies are followed. Serious adverse events are recorded and those possibly related to the study procedure are reported to the principal investigator within $24 \mathrm{~h}$

\section{Dissemination}

The results will be published in international scientific journals. The outcomes of the trial will be presented in international congresses.

\section{Discussion}

There are unresolved questions concerning the ideal extent of ESS in patients with severe CRSwNP. A systemic Cochrane review concluded that there are no trials comparing simple polypectomy and extensive ESS, which would have met their inclusion criteria ${ }^{(24)}$.

This randomized controlled trial compares safety and efficacy of limited and extended ethmoidectomy in the treatment of severe CRSwNP. We will monitor general and disease-spesific HRQoL in CRS and asthma, sense of smell, endoscopic nasal polyp score, lung function, absenteeism and days of decreased productivity during work, as well as the use of treatment resources including their economic aspects in order to assess the rational use of surgical resources. Adverse events of treatments will be monitored throughout the study.

\section{Trial status}

The trial started in November 2018. We are currently recruiting patients. The anticipated completion of recruitment is beginning of year 2022 .

\section{Authorship contribution}

PV is the principal investigator in the Helsinki University Hospital and coordinating investigator with regard to all participating sites. ML, PV and STS prepared the manuscript. All authors read and approved the final manuscript.

\section{Acknowledgments}

Not applicable.

\section{Funding}

The study was supported in part by research grants from Finnish Society of Allergology and Immunology, the Jane and Aatos Erkko Foundation, Paulo Foundation, State funding for university-level health research (TYH2019322), the Tampere Tuberculosis Foundation, the Väinö and Laina Kivi Foundation, Yrjö Jahnsson Foundation, Foundation of the Finnish Anti-Tuberculosis Association.

\section{Ethics approval and consent to participate} Not applicable. 


\section{Consent for publication}

Not applicable.

\section{Availability of data and materials}

Not applicable.

\section{Conflict of interest}

STS reports consultancies for AstraZeneca, ERT, Novartis, Sanofi Pharma, and Roche Products and a grant of GSK, outside the submitted work. WF reported consultancies for Sanofi and GSK outside the submitted work. JSA reports consultancy for Sanofi outside the submitted work. All other authors declare no con-

flicts of interest.

\section{Orcid iD}

Markus Lilja (0000-0003-1781-1718), Paula Virkkula (0000-00034224-5201), Sari Hammarén-Malmi (Undefined), Anu Laulajainen-Hongisto (0000-0001-5109-6944), Lena Hafrén (0000-00024968-4357), Paula Kauppi (0000-0002-1065-330x), Johanna Sahlman (Undefined), Wytske Fokkens (0000-0003-4852-229x), Sietze Reitsma (Undefined), Sanna Toppila-Salmi (0000-00030890-6686).

\section{References}

1. Hastan D, Fokkens WJ, Bachert C, et al. Chronic rhinosinusitis in Europe--an underestimated disease. A GA(2)LEN study. Allergy. 2011 Sep;66(9):1216-1223

2. Dietz de Loos D, Lourijsen ES, Wildeman MAM, et al. Prevalence of chronic rhinosinusitis in the general population based on sinus radiology and symptomatology. J Allergy Clin Immunol. 2019 Mar;143(3):1207-1214

3. Fokkens WJ, Lund VJ, Hopkins C, et al. European Position Paper on Rhinosinusitis and Nasal Polyps 2020. Rhinology. 2020 Feb 20;58(Suppl S29):1-464.

4. Lourijsen ES, Fokkens WJ, Reitsma S. Direct and indirect costs of adult patients with chronic rhinosinusitis with nasal polyps. Rhinology. 2020 Jun 1;58(3):213-217.

5. Masterson L, Tanweer F, Bueser T, Leong P. Extensive endoscopic sinus surgery: does this reduce the revision rate for nasal polyposis? Eur Arch Otorhinolaryngol. 2010 Oct;267(10):1557-1561.

6. Jankowski R, Pigret D, Decroocq F, Blum A, Gillet P. Comparison of radical (nasalisation) and functional ethmoidectomy in patients with severe sinonasal polyposis. A retrospective study. Rev Laryngol Otol Rhinol (Bord). 2006;127(3):131-140

7. Chen FH, Deng J, Hong HY, et al. Extensive versus functional endoscopic sinus surgery for chronic rhinosinusitis with nasal polyps and asthma: A 1-year study. Am J Rhinol Allergy. 2016 Mar-Apr;30(2):143-148.

8. Stammberger $\mathrm{H}$, Posawetz W. Functional endoscopic sinus surgery. Concept, indications and results of the Messerklinger technique. Eur Arch Otorhinolaryngol. 1990;247(2):63-76.

9. Zhang L, Zhang Y, Gao Y, et al. Long-term outcomes of different endoscopic sinus surgery in recurrent chronic rhinosinusitis with nasal polyps and asthma. Rhinology. 2020
Apr 1;58(2):126-135.

10. Toppila-Salmi S, Rihkanen H, Arffman M, Manderbacka K, Keskimaki I, Hytonen $M L$. Regional differences in endoscopic sinus surgery in Finland: a nationwide register-based study. BMJ Open. 2018 Oct 18:8(10):e022173-2018-022173.

11. Browne JP, Hopkins C, Slack R, et al. Healthrelated quality of life after polypectomy with and without additional surgery. Laryngoscope. 2006 Feb;1 16(2):297-302.

12. Suzuki S, Yasunaga $H$, Matsui $H$, Fushimi K, Kondo K, Yamasoba T. Complication rates after functional endoscopic sinus surgery: analysis of 50,734 Japanese patients. Laryngoscope. 2015 Aug;125(8):1785-1791.

13. Thomas AJ, Smith KA, Newberry Cl, et al. Operative time and cost variability for functional endoscopic sinus surgery. Int Forum Allergy Rhinol. 2019 Jan;9(1):23-29.

14. Fokkens WJ, Lund VJ, Mullol J, et al. European Position Paper on Rhinosinusitis and Nasal Polyps 2012. Rhinol Suppl. 2012 Mar;23:3 p preceding table of contents, 1-298.

15. Hopkins C, Gillett S, Slack R, Lund VJ, Browne JP. Psychometric validity of the 22-item Sinonasal Outcome Test. Clin Otolaryngol. 2009 Oct;34(5):447-454

16. Koskinen A, Hammarén-Malmi S, Myller J, et al. Translation, cross-cultural adaptation, and validation of the sino-nasal outcome test (snot)-22 for Finnish patients. Eur Arch Otorhinolaryngol. 2021;278:405-410.

17. Sintonen $H$. The $15 D$ instrument of healthrelated quality of life: properties and applications. Ann Med. 2001 Jul;33(5):328-336.

18. Alanne S, Roine RP, Rasanen P, Vainiola $\mathrm{T}$, Sintonen $\mathrm{H}$. Estimating the minimum important change in the 15D scores. Qual Life Res. 2015 Mar;24(3):599-606.

19. Psaltis AJ, Li G, Vaezeafshar R, Cho KS, Hwang $\mathrm{PH}$. Modification of the LundKennedy endoscopic scoring system improves its reliability and correlation with patient-reported outcome measures. Laryngoscope. 2014 Oct;124(10):2216-2223.

20. Gevaert P, Omachi TA, Corren J, et al. Efficacy and safety of omalizumab in nasal polyposis: 2 randomized phase 3 trials. J Allergy Clin Immunol. 2020 Sep;146(3):595605.

21. Hellings PW, Fokkens WJ, Akdis C, et al. Uncontrolled allergic rhinitis and chronic rhinosinusitis: where do we stand today? Allergy. 2013 Jan;68(1):1-7.

22. Bouwmans C, Hakkaart-van Roijen L, Koopmanschap M, Krol M, Severens $H_{\text {, }}$ Brouwer W. Handleiding iMTA Productivity Cost Questionnaire (iPCQ). Rotterdam: iMTA, Erasmus Universiteit. 2013.

23. Bouwmans C, Hakkaart-van Roijen L, Koopmanschap M, Krol M, Severens J, Brouwer W. Handleiding iMTA Medical Cost Questionnaire (iMCQ). Rotterdam: iMTA, Erasmus Universiteit Rotterdam. 2013.

24. Sharma R, Lakhani R, Rimmer J, Hopkins C. Surgical interventions for chronic rhinosinusitis with nasal polyps. Cochrane Database Syst Rev. 2014 Nov 20;(11):CD006990

Markus Lilja MD, PhD

Department of Otorhinolaryngology

Kasarmikatu 11-13

P.O. Box 263

00029 HUS

Helsinki

Finland

Tel: +35894711

E-mail: markus.lilja@hus.fi

ISSN: 2589-5613 / ( 2021 The Author(s). This work is licensed under a Creative Commons Attribution 4.0 International License. The images or other third party material in this article are included in the article's Creative Commons license, unless indicated otherwise in the credit line; if the material is not included under the Creative Commons license, users will need to obtain permission from the license holder to reproduce the material. To view a copy of this license, visit http://creativecommons.org/ licenses/by/4.0/ 